\title{
Interference and Conflict Simulation of Left-turning Vehicle and Pedestrian Based on Cellular Automation
}

\author{
Xueyuan Wang ${ }^{a}{ }^{*}$, Xingqiang Zhang ${ }^{b}$, Pan Chen ${ }^{c}$ \\ School of Traffic and Transportation, Beijing Jiaotong University, Beijing 100044, China \\ a16120893@bjtu.edu.cn, bzhangxq@bjtu.edu.cn, c546175775@qq.com \\ *corresponding author
}

\begin{abstract}
In order to analyze microscopic operating law of intersection mixed traffic flow, a coupled cellular model of the vehicle VDR model and the one-way pedestrian Blue model was presented. Based on the definition of pedestrian $\rightarrow$ vehicle interference area, vehicle $\rightarrow$ pedestrian interference area and the crossing behaviors of left-turning vehicle and pedestrian, the interference and conflict rules between left-turning vehicle and pedestrian at intersection were formulated. Finally, the relationships of traffic flow states, variation trend, and arrival rate were simulated to study the mixed traffic characteristics of left-turning vehicle and pedestrian.
\end{abstract}

Keywords: conflict interference; VDR-Blue model; arrival rate; traffic flow volume

\section{Introduction}

The reasonable establishment of vehicle and pedestrian traffic simulation model at urban intersection can reappear dynamically the micro operation law of intersection mixed traffic and provide theoretical basis for the intersection signal control and traffic organization optimization. Gao Liping(2011) $)^{[1]}$, Ma Wanjing(2015) ${ }^{[2]}$ et al. have researched the intersection interference mechanisms and crossing behaviors of vehicle, non-motor vehicle and pedestrian. In the aspect of cellular automata simulation, Blue(1998) ${ }^{[3]}$, Ren $\operatorname{Gang}(2012)^{[4]}$ et al. have studied and established the corresponding cellular automaton model of pedestrian flow from the aspects of pedestrian flow status and pedestrian evacuation; Zhang Xingqiang(2012) ${ }^{[5]}$, Sun Ze(2012) ${ }^{[6]}$, et al. have established the cellular automaton model of mixed vehicle-pedestrian traffic flow from the micro level and analyzed the characteristics of mixed traffic between vehicle and pedestrian. This paper established a crossing behaviors cellular automaton model of left-turning vehicle and pedestrian, presented the VDR-Blue coupling cellular automaton model based on VDR model and Blue model, revealed the interference mechanism of intersection vehicle-pedestrian mixed traffic flow.

\section{The Operation Rules of Vehicle and Pedestrian}

This paper regards that the operation of left-turning vehicle meets VDR model and the pedestrian meets one-way Blue model. In model, left-turning roadway is denoted as Lane, Lane ${ }_{1}$ is the entrance roadway, its length is $\mathrm{L}_{1}$; Lane $_{\mathrm{N}}$ is the Vehicle $\rightarrow$ pedestrian interference roadway and its 
length is $L_{N}$; Lane ${ }_{2}$ is the exit roadway and length is $L_{2}$; pedestrian crosswalk is denoted as $Z$, its width is $\mathrm{L}_{\mathrm{rk}}$ and length is $\mathrm{L}_{\mathrm{rc}}$; pedestrian $\rightarrow$ Vehicle interference area is denoted as $\mathrm{M}$ with length $\mathrm{L}_{\mathrm{M}}$; conflict area is denoted as $\mathrm{C}$, its length is $\mathrm{L}_{\mathrm{c}}$ and width is $\mathrm{L}_{\mathrm{rk}}$ which is equal to the width of pedestrian crosswalk. $\mathrm{X}$ is the boundary line for vehicle entering the conflict area $\mathrm{C}$ (Figure.1).

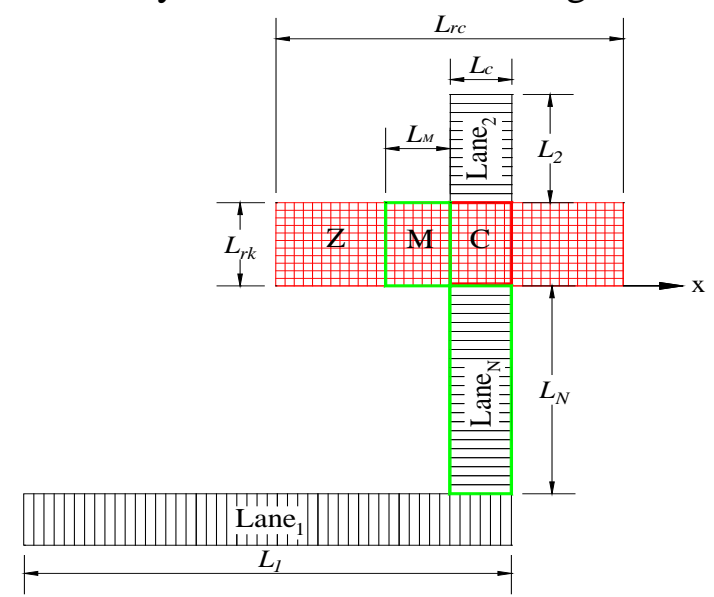

Fig.1 Traffic Cell Spatial Division

\subsection{The Rules of Vehicle Operating on the Road}

1. Arrival and departure rules of left-turning vehicle

$$
\text { if } X_{\text {Last }}(t)>V_{\max }^{1} \text {, Then } \eta(t)<\alpha_{V} \Rightarrow X_{\text {New }}(t)=\min \left(X_{\text {Last }}(t)-V_{\max }^{1}, V_{\max }^{1}\right) ; \quad X_{\text {First }}(t)>L_{1}+L_{N}+L_{r k}+L_{2}
$$

2. Acceleration and deceleration rules

$$
V_{n}(t+1)=\min \left[V_{n}(t)+1, V_{\max }^{1}\right], V_{n}(t+1)=\min \left[V_{n}(t+1), d_{n}(t)\right], d_{n}(t)=X_{n+1}(t)-X_{n}(t)-l_{c}
$$

3. Stochastic slowing down rules

$$
\text { flag }=\left\{\begin{array}{ll}
1 & f_{1}=0.75 \\
0 & f_{2}=0.2
\end{array}, V_{n}(t+1)=\min \left[V_{n}(t+1)-1,0\right]\right.
$$

4. Vehicle movement rules

$$
X_{n}(t+1)=X_{n}(t)+V_{n}(t+1)
$$

where $X_{\text {Last }}(t)$ is the position of the last vehicle on the entrance roadway Lane ${ }_{1}$ at time $t ; V_{\max }^{1}$ is the maximum speed of vehicle on the road; $\eta(t)$ is the uniform random number among $[0,1] ; \alpha_{V}$ is the arrival rate of vehicle; $X_{\text {New }}(t)$ is the position of a new vehicle on Lane ${ }_{1}$ at time t; $X_{\text {First }}(t)$ is the position of the first vehicle on the exit roadway Lane 2 at time $t ; V_{n}(t)$ is the speed of vehicle $\mathrm{N}$ at time $\mathrm{t} ; d_{n}(t)$ is the number of vacant cells between vehicle $\mathrm{N}$ and its front one at time $\mathrm{t} ; X_{n}(t)$ is the position of vehicle $\mathrm{N}$ at time $\mathrm{t} ; f_{1}, f_{2}$ are the stochastic slowing down probability.

\subsection{The Rules of Pedestrian Operating in the Crosswalk ${ }^{[3]}$}

if $n_{z_{i, j}}=n_{z_{i, j}}^{j-1}=n_{z_{i, j}}^{j+1}$, the probability of pedestrian walking in current crosswalk, the left crosswalk and the right crosswalk is $80 \%, 10 \%, 10 \%$ respectively.

if $\max \left(n_{z_{i, j}}, n_{z_{i, j}}^{j-1}, n_{z_{i, j}}^{j+1}\right)=n_{z_{i, j}}, n_{z_{i, j}}^{j-1}$ or $\max \left(n_{z_{i, j}}, n_{z_{i, j}}^{j-1}, n_{z_{i, j}}^{j+1}\right)=n_{z_{i, j}}, n_{z_{i, j}}^{j+1}$, the probability of pedestrian walking in current crosswalk, the left crosswalk and the right crosswalk is $80 \%$, 20\% respectively.

if $\max \left(n_{z_{i, j}}, n_{z_{i, j}}^{j-1}, n_{z_{i, j}}^{j+1}\right)=n_{z_{i, j}}^{j-1}=n_{z_{i, j}}^{j+1}$, the probability of pedestrian walking in the left crosswalk and the right crosswalk is $50 \%$, 50\% respectively.

where $Z_{1, j}$ is the first column cells of the crosswalk $Z ; n_{z_{i, j}}, n_{z_{i, j}}^{j-1}, n_{z_{i, j}}^{j+1}$ is the number of vacant cells which in front of the cell $(i, j)$, in front of the right cell adjacent $(i, j)$, and in front of the left cell adjacent $(i, j)$ respectively. 


\section{VDR-Blue Rules}

When the interference between pedestrian flow and left-turning flow occurring, pedestrian decides whether to enter into the conflict area is affected by the distance between vehicle and conflict area and the speed of vehicle at that moment. The probability of left-turning vehicle entering into the conflict area is calculated based on the Logistic regression model which concluded by Qian Dalin ${ }^{[7]}$.

$$
P_{P \rightarrow V}=\frac{e^{2.526+0.905 G_{V}-1.530 V}}{1+e^{2.526+0.905 G_{V}-1.530 V}}, \quad P_{V \rightarrow P}=\frac{1}{1+e^{2.953-0.856 G_{c}-1.319 V+3.562 v+0.117 G_{r}}}
$$

where $P_{P \rightarrow V}$ is the probability of pedestrian crossing left-turning vehicle; $P_{V \rightarrow P}$ is the probability of left-turning vehicle crossing pedestrian; $G_{V}$ is the distance between vehicle and conflict area, $m ; G_{c}$ is the current gap provided by pedestrian, $m ; G_{r}$ is the reference gap provided by pedestrian, $m$.

The vehicle in Vehicle $\rightarrow$ Pedestrian interference area which closest to the boundary line $\mathrm{X}$ of conflict area is denoted as vehicle $l$, its position is $X_{l}$; the vehicle in conflict area which closest to the boundary line $\mathrm{X}$ of conflict area is denoted as vehicle $r$, its position is $X_{r}$. The state of the cell $s_{n}(t)$ is the distance between pedestrian $\mathrm{n}$ and conflict area at time $\mathrm{t}, \boldsymbol{V}_{n}(t)$ is the speed of pedestrian $\mathrm{n}$ at time $\mathrm{t}$. $\mathrm{C}$ in conflict area is:

$$
\begin{aligned}
& C_{i, j}(t)= \begin{cases}0 & C \text { vacant } \\
1 & \text { C occupied by pedestrian } \\
2 & C \text { occupied by vehicle }\end{cases} \\
& i \in\left(\frac{1}{2} L_{r c}+1, \frac{1}{2} L_{r c}+L_{c}\right) ; j \in\left(L_{1}+L_{N}+1, L_{1}+L_{N}+L_{r k}\right)
\end{aligned}
$$

The rules of interference between left-turning vehicle and pedestrian in the intersection are:

1. if $X_{l} \leq L_{1}$, vehicle $l$ doesn't in the Vehicle $\rightarrow$ Pedestrian interference area at time $\mathrm{t}$ and operating on VDR rule, pedestrian operating rules are divided into:

1) if $\mathrm{s}_{n}(t)>v_{n}(t)$, vehicle operating on VDR rule, pedestrian operating on Blue rule.

2) if $\mathrm{s}_{n}(t) \leq v_{n}(t)$, pedestrian can reach conflict area $\mathrm{C}$ at time $\mathrm{t}+1$.

(1) if $C(t)=0$ or $C(t)=1$, pedestrian entering into $C$ on Blue rule, vehicle operating on VDR rule.

(2) if $C(\mathrm{t})=2$, conflict area is occupied by vehicle $r$. Pedestrian behavior at time $\mathrm{t}+1$ is divided into the following circumstances:

(a) if $L_{1}+L_{N}<X_{r} \leq L_{1}+L_{N}+l_{c}$, the pedestrian who can enter into C stop walking at time $\mathrm{t}+1$.

(b) if $L_{1}+L_{N}+l_{c}<X_{r}<L_{1}+L_{N}+L_{r k}+l_{c}$ :

When $L_{1}+L_{N}+1 \leq j<X_{r}-l_{c}+1$, the pedestrian between the boundary line $\mathrm{X}$ and the rear of vehicle entering into C; When $X_{r}-l_{c}+1 \leq j<L_{1}+L_{N}+L_{r k}$, the pedestrian who can enter into C at time $\mathrm{t}+1$ stop walking, the other pedestrian operating on Blue rule.

2. if $L_{1}<X_{l}<L_{1}+L_{N}-V_{n}$, vehiclel in interference area can't reach conflict area C at time $\mathrm{t}+1$. Vehicle operating on VDR rule, pedestrian operating rules are divided into:

1) if $s_{n}(t)>v_{n}(t)$, vehicle operating on VDR rule, pedestrian operating on Blue rule.

2) if $\mathrm{s}_{n}(t) \leq v_{n}(t)$ :

(1) if $C(t)=0$ or $C(t)=1$, pedestrian can make decisions whether or not to cross left-turning vehicle, the probability of pedestrian entering into $\mathrm{C}$ is $P_{P \rightarrow V}^{1}$. 
(2) if $C(\mathrm{t})=2$ :

(a) if $L_{1}+L_{N}<X_{r} \leq L_{1}+L_{N}+l_{c}$, the pedestrian who can enter into $C$ at time $\mathrm{t}+1$ stop walking, the other pedestrian operating on Blue rule.

(b) if $L_{1}+L_{N}+l_{c}<X_{r}<L_{1}+L_{N}+L_{r k}+l_{c}$ :

When $L_{1}+L_{N}+1 \leq j<X_{r}-l_{c}+1$, the probability of pedestrian entering into $\mathrm{C}$ is $P_{P \rightarrow V}^{2}$;

When $X_{r}-l_{c}+1 \leq j<L_{1}+L_{N}+L_{r k}$, the pedestrian who can enter into C stop walking at time $\mathrm{t}+1$, the other pedestrian operating on Blue rule.

3. if $X_{l}>L_{1}+L_{N}-V$, vehiclel in interference area can reach conflict area $\mathrm{C}$ at time $\mathrm{t}+1$ :

1) if $C(\mathrm{t})=0$ :

(1) if $s_{n}(t)>L_{M}$, vehiclel entering into conflict area $C$ at time $t+1$ and operating on VDR rule, pedestrian operating on Blue rule.

(2) if $s_{n}(t) \leq v_{n}(t)$, the probability of pedestrian having priority to enter into $C$ is $P_{P \rightarrow V}^{3}$, vehicle deceleration operation to $\mathrm{C}$.

(3) if $s_{n}(t)>v_{n}(t)$, vehicle $l$ can make decisions whether or not to cross pedestrian, the probability of vehicle entering into $\mathrm{C}$ is $P_{V \rightarrow P}^{1}$, pedestrian operating on Blue rule.

2) if $C(\mathrm{t})=1$ :

(1) if $s_{n}(t) \leq v_{n}(t)$, the probability of pedestrian following to enter into $C$ is $P_{P \rightarrow V}^{3}$.

(2) if $s_{n}(t)>v_{n}(t)$, the pedestrian who can't enter into $C$ at time $t+1$ operating on Blue rule.

3) if $C(\mathrm{t})=2$ :

(1) if $L_{1}+L_{N}<X_{r} \leq L_{1}+L_{N}+l_{c}$, the pedestrian who can enter into $\mathrm{C}$ at time $\mathrm{t}+1$ stop walking, the other pedestrian operating on Blue rule, Vehicle operating on VDR rule.

(2) if $L_{1}+L_{N}+l_{c}<X_{r}<L_{1}+L_{N}+L_{r k}+l_{c}$ :

(a) if $S_{n}(t)>L_{M}$, vehicle operating on VDR rule, pedestrian operating on Blue rule.

(b) if $s_{n}(t) \leq v_{n}(t)$, the pedestrian who can enter into C stop walking at time $\mathrm{t}+1$.

(c) if $s_{n}(t)>v_{n}(t)$, probability of vehicle entering $\mathrm{C}$ is $P_{V \rightarrow P}^{2}$, pedestrian operating on Blue rule.

\section{Model Simulation}

In the simulation, the size of vehicle cell is $0.5 \times 3 \mathrm{~m}$, each vehicle occupies $l_{c}=12$ cells, and the size of pedestrian cell is $0.5 \times 0.5 m . \mathrm{L}_{1}=406, \mathrm{~L}_{\mathrm{N}}=24, \mathrm{~L}_{2}=360, L_{r k}=10, \mathrm{~L}_{\mathrm{rc}}=36, \mathrm{~L}_{\mathrm{M}}=6, \mathrm{~L}_{\mathrm{c}}=6$ and $\mathrm{L}_{\mathrm{rk}}=10$ (Figure.1). The simulation step length is 1s, the simulation uses the 10001-20000 step traffic flow data and analyzes the relationship between flow volume $Q_{v}, Q_{p}$ and arrival rate $\alpha_{v}, \alpha_{p}$. 
4.1 Relationship between $Q_{v}, Q_{p}$ and $\alpha_{v}, \alpha_{p}$

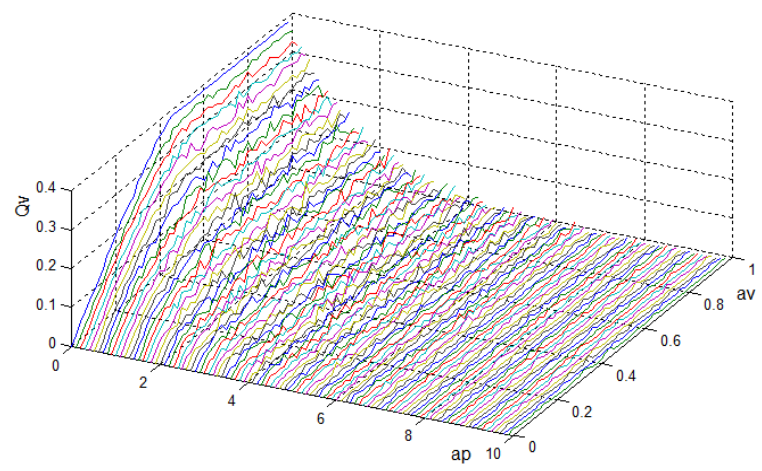

Fig.2 Relationship diagram of $Q_{v}-\alpha_{v}-\alpha_{p}$

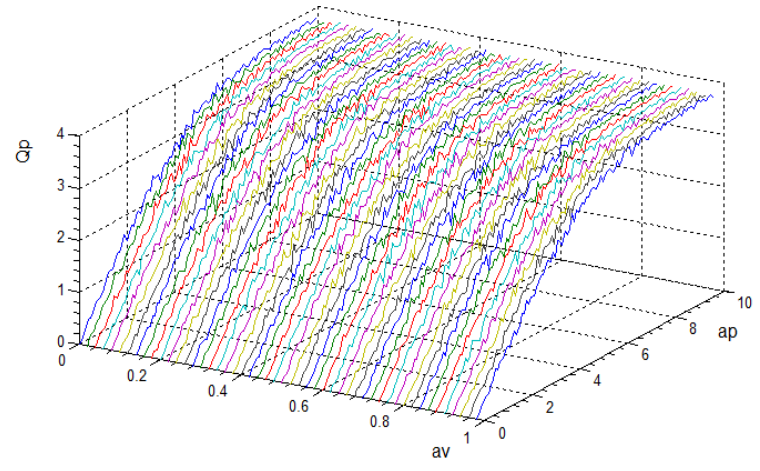

Fig.3 Relationship diagram of $Q_{p}-\alpha_{p}-\alpha_{v}$

Figure.2 shows the relationship between vehicle flow volume $Q_{v}$ and pedestrian arrival rate $\alpha_{p}$, vehicle arrival rate $\alpha_{v}$. When $\alpha_{p}<4.6$, there is a critical vehicle arrival rate $\alpha_{v}^{c}$. When $\alpha_{v}<\alpha_{v}^{c}, Q_{v}$ is approximately linear with $\alpha_{v}$; When $\alpha_{v} \geq \alpha_{v}^{c}, Q_{v}$ reached saturation and fluctuated slightly. When $\alpha_{p}=0, \alpha_{v}^{c}=0.46$, vehicle saturation volume $Q_{v}=0.384$; when $0<\alpha_{p}<2.0, Q_{v}$ decrease greatly; when $2.0 \leq \alpha_{p}<4.6, Q_{v}$ decrease slowly; when vehicle flow volume is close to zero, vehicle will stop and wait for pedestrian to pass through the crosswalk.

Figure.3 shows the relationship between pedestrian flow volume $Q_{p}$ and pedestrian arrival rate $\alpha_{p}$, vehicle arrival rate $\alpha_{v}$. There is a critical pedestrian arrival rate $\alpha_{p}^{c}=7.5$, when $\alpha_{p}<\alpha_{p}^{c}, Q_{p}$ is approximately linear with $\alpha_{p}$; when $\alpha_{p} \geq \alpha_{p}^{c}, Q_{p}$ reached saturation and fluctuated slightly. $\alpha_{v}$ has little influence on pedestrian saturation volume $Q_{p}$ and pedestrian critical arrival rate $\alpha_{p}^{c}$, it has a little fluctuation around $Q_{p}=3.7, \alpha_{p}^{c}=7.5$, vehicle has less interference to pedestrian.

\subsection{The Traffic Flow Phase of Left-turning Vehicle and Pedestrian}

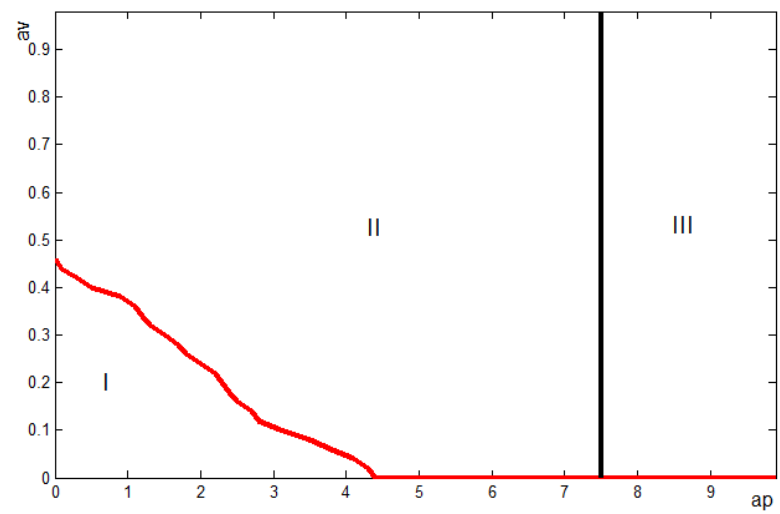

Fig.4 Phase diagram of Traffic Flow

The phase diagram of vehicle and pedestrian traffic flow are shown in Figure.4. With the increase of $\alpha_{p}, \alpha_{v}^{c}$ gradually decreased to zero, but $\alpha_{p}^{c}$ doesn't vary with the change of $\alpha_{v}$, which shows that pedestrian is less disturbed by vehicle. The traffic flow is divided into three phases, in phase I, both vehicle and pedestrian flow are free flow; in phase II, vehicle flow reaches a steady state and the pedestrian flow is still free flow, when $\alpha_{p} \geq 4.6$, vehicle flow volume is close to zero; in phase III, both vehicle and pedestrian flow are steady flow, and the vehicle flow volume is zero. 


\section{Conclusions}

This paper established the VDR-Blue cellular automaton model to analyze the mixed traffic flow characteristics between left-turning vehicle and pedestrian. The results of simulation analysis show that there is a critical arrival rate which makes the vehicle flow volume and pedestrian flow volume having phase transition from free flow to saturated flow, the saturation volume of vehicle and pedestrian is mainly affected by pedestrian arrival rate;. and almost unaffected by vehicle arrival rate; the mixed traffic flow of left-turning vehicle and pedestrian can be divided into 3 phases: both free flow; vehicle flow is steady flow and pedestrian flow is free flow; both steady flow.

\section{References}

[1] Gao Liping, FU Chunjie, HE Huijun, et al. Delay Model of Ped-Veh System Based on Pedestrian Crossing Signalized Roads[J]. Journal of Transportation Systems Engineering and Information Technology, 2011, 11(1):14-21.

[2] Ma Wanjing, YE Xinchen, LIAO Dabin, et al. Distribution Characteristic of Accepted Gap and Rejected Gap for Vehicles Crossing Stop- Controlled Intersections[J]. China Journal of Highway and Transport, 2015, 28(4):86-93.

[3] Blue V, Adler J. Emergent Fundamental Pedestrian Flows from Cellular Automata Micro-simulation [J].Transportation Research Record, 1998, 1644(1):29-36.

[4] Ren Gang, LU Lili, WANG Wei. Modeling bi-direction pedestrian flow by cellular automata and complex network theories[J]. Acta Phy. Sin., 2012,61(14):255-264.

[5] Zhang Xingqiang, WANG Ying, HU Qinghua. Research and simulation on cellular automaton model of mixed traffic flow at intersection[J]. Acta Phy. Sin. 2014,63(1):90-97.

[6] Sun Ze, JIA Bin, LI Xingang. The study of the interference between pedestrians and vehicles based on cellular automaton model[J]. Acta Phy. Sin. 2012, 61(10):76-83.

[7] Qian Dalin, LI Shanshan, CHEN Xiaohong. Microscopic simulation and application of pedestrian and bicycle traffic[M]. Beijing: China Communications Press, 2011:53-59. 